\title{
A Review of Power Electronic Parametric Fault Diagnosis Methods
}

\author{
Wan, Zhisong; Lin, Qiongbin; Qiao, Feng; Zhang, Zhe; Zong, Yi; Su, Xianjin
}

Published in:

Proceedings of 4th International Conference on Intelligent Green Building and Smart Grid

Link to article, DOI:

10.1109/igbsg.2019.8886330

Publication date:

2019

Document Version

Peer reviewed version

Link back to DTU Orbit

Citation (APA):

Wan, Z., Lin, Q., Qiao, F., Zhang, Z., Zong, Y., \& Su, X. (2019). A Review of Power Electronic Parametric Fault Diagnosis Methods. In Proceedings of 4th International Conference on Intelligent Green Building and Smart Grid (pp. 448-451). IEEE. https://doi.org/10.1109/igbsg.2019.8886330

\section{General rights}

Copyright and moral rights for the publications made accessible in the public portal are retained by the authors and/or other copyright owners and it is a condition of accessing publications that users recognise and abide by the legal requirements associated with these rights.

- Users may download and print one copy of any publication from the public portal for the purpose of private study or research.

- You may not further distribute the material or use it for any profit-making activity or commercial gain

- You may freely distribute the URL identifying the publication in the public portal 


\section{A Review of Power Electronic Parametric Fault Diagnosis Methods}

\author{
$1^{\text {st }}$ Zhisong Wan \\ College of Electrical Engineering and \\ Automation \\ Fuzhou University \\ Fuzhou, China \\ n170127052@,fzu.edu.cn \\ $4^{\text {th }}$ Zhe Zhang \\ Department of Electrical Engineering \\ Technical University of Denmark \\ 2800 Kgs. Lyngby, Denmark \\ zz@elektro.dtu.dk
}

\author{
$2^{\text {nd }}$ Qiongbin Lin \\ College of Electrical Engineering and \\ Automation \\ Fuzhou University \\ Fuzhou, China \\ qiongbinlin@fzu.edu.cn \\ $5^{\text {th }}$ Yi Zong \\ Department of Electrical Engineering \\ Technical University of Denmark \\ 2800 Kgs. Lyngby, Denmark \\ yizo@elektro.dtu.dk
}

\author{
$3^{\text {rd }}$ Feng Qiao \\ College of Electrical Engineering and \\ Automation \\ Fuzhou University \\ Fuzhou, China \\ qiaofeng1122@live.com
}

$6^{\text {th }}$ Xianjin $\mathrm{Su}$

Kehua Hengsheng Co., Ltd.

Xiamen, China

suxianjin@,kehua.com

\begin{abstract}
This paper attempts to present a concise review of the research done around parametric fault diagnosis methods for power electronic circuits, including model-based, knowledge-based, signal-based and other emerging fusion methods. Comments on the advantages and constraints of various methods, are also given. Finally, combined with the bottlenecks faced by the current parametric fault diagnosis field of power electronic circuits, the new trends of future research in this field are discussed.
\end{abstract}

Keywords—power electronics, parametric fault diagnosis

\section{INTRODUCTION}

With the rapid development of the social economy, power electronic devices have been widely used in various industries. Especially in the field of new energy, the compound average growth rate of electric, full-hybrid electric, mild-hybrid electric, and plug-in-hybrid EVs will reach 28\% from 2015 through 2022 [1]. In China, new energy power generation is developing rapidly, with installed capacity and power generation ranking first in the world. By the end of 2018, the total installed capacity of new energy power generation had exceeded 350 million $\mathrm{kW}$, and it increased of $21.9 \%$ year-on-year [2].

In order to meet the needs of users in these fields, a variety of new power electronics products is emerging and the structure is becoming more complex. This will inevitably lead to increase significantly the possibility of new faults in the equipment, making the diagnosis more difficult. According to the degree of change of the macro performance of the circuit caused by the failure of the power electronic device, the fault can be divided into two categories [3]: structural failures and parametric failures. Structural faults, also known as hard faults, refer to a type of fault that causes a circuit topology change due to a serious failure such as an open circuit or a short circuit of the power electronics. Hard faults are sudden and often lead to drastic changes in circuit functions, which can easily lead to catastrophic consequences. Parametric faults, also known as soft faults, are fault types in which electronic components are offset as their performance degrades. Soft faults have a gradual change, which does not cause a sudden loss of circuit function, but it will lead to changes in system performance. If it is not processed effectively, it will eventually become a catastrophic failure. Therefore, an in-depth study of power electronics early parametric fault diagnosis methods is not only conducive to detect and eliminate timely potential parameter faults of equipment, but also fault diagnosis and redundant design to reduce system failure rate. To improve equipment reliability, these are of great practical significance.

The fault diagnosis technology of power electronic systems has developed rapidly in the past decade, but it mainly focused on structural failures. In recent years, with the complexity and modularization of power electronic systems, parametric fault diagnosis methods for the degradation of capacitors and switching tubes have also been continuously studied. Based on these studies, this paper summarizes parametric fault diagnosis methods of power electronic converters, including model-based, knowledgebased, signal-based and other emerging fusion methods, and analyzes simultaneously the advantages and constraints of various methods, and finally combined with the current bottlenecks in the field of parametric fault diagnosis of power electronic circuits, explored new trends in future research in this field.

\section{RESEARCH StATUS OF POWER ELECTRONIC PARAMETRIC FAULT DiAgNosis METHODS}

A. Model-based fault diagnosis methods

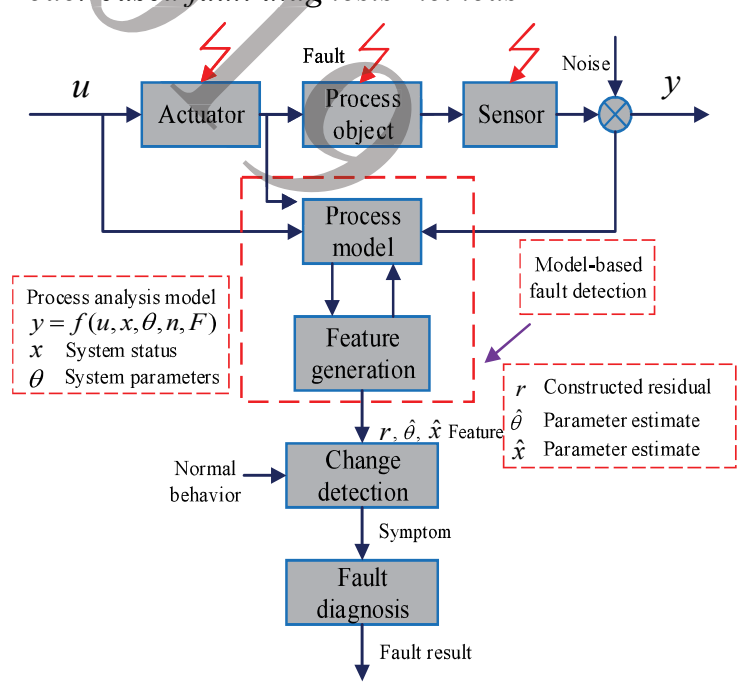

Fig. 1 Schematic of model-based parametric fault diagnosis

Early power electronic parametric fault diagnosis methods were based on analytical models. The model-based method is a technique of comparing the measured value of the object to be diagnosed with the estimated value of the 
system established by the mathematical model to generate a residual, and using the residual for fault analysis and processing. The principle is shown in Fig. 1. According to the actuator and the sensor, through the analytical model of the establishment process, the residual value is used to detect the change between the measured value and the estimated value, thereby achieving the purpose of fault diagnosis.

According to the difference of residual forms, it can be subdivided into parameter estimation approach and state estimation approach.

For the parameter estimation and identification approach, in [4], firstly it starts from the basic physical characteristics of the power electronic circuit, and which is represented by the hybrid system model. Then the state and parameters of the hybrid electronic power circuit model are estimated by strong tracking filter, and the modified Bayes algorithm is used to make real-time fault diagnosis. A fault diagnosis method based on online model is proposed in [5]. For the aging of the device, the particle swarm optimization algorithm is used to identify the device parameters online.

In terms of estimating capacitors equivalent series resistance (ESR), since capacitors usually determine the overall life of power electronic converters, they are the main source of faults. A new technology to detect faults in capacitors in Boost converters is proposed by [6], and it can also implement real-time Online diagnosis. This technology is based on a double estimate of ESR and capacitance, which improves diagnostic reliability and predicts maintenance. In [7], compensates for ESR by using a cost-effective temperature sensing circuit and a thermistor to obtain a more accurate estimate. From the estimated ESR values in the experiment, the deterioration of the electrolytic capacitor can be diagnosed effectively and accurately.

In the research of state estimation approach, [8] reviewed the power electronic reliability modeling method, from the device structure to the thermoelectric and thermomechanical viewpoints, thus developing the state monitoring technology of power electronics. This technology usually uses a priori knowledge-based model to detect faults in the current mode and state of power electronic converters, providing an effective means to improve reliability.

Although the fault diagnosis technology based on analytical model is researched earlier and applied in process control systems in some industries, the current situation in the literature is: due to strong interference and the nonlinearity of the object of the diagnosed power electronic system, the fault diagnosis method of the analytical model still has many blanks in a wide variety of power conversion systems.

\section{B. Knowledge-based fault diagnosis methods}

As mentioned above, the modeling of nonlinear power electronic systems is very difficult, and there are not many means for estimating the parameters or estimating the state of fault diagnosis. Especially when the model of the monitored system is uncertain and the statistical characteristics of the noise are not ideal, it is more difficult to get a more accurate parameter estimate or state estimate. The knowledge-based diagnosis method does not rely on the control system analysis model, thus avoiding the difficulty of extracting the mathematical model of the object. At present, knowledgebased methods for power electronic system fault diagnosis include neural network approach, fuzzy inference approach, and pattern recognition approach.

In the research of neural network approach, [9] uses the closed-loop controlled DC-DC conversion circuit as a diagnostic example. Under different working conditions, it simulates the hard and soft faults in the main circuit based on simulation experiments and physical experiments. At the same time, aiming at the shortcomings of back-propagation neural network (BPNN) apt to fall into local minimum value, an improved method for initial weight and threshold optimization of BPNN using thought evolution algorithm is proposed. Then in [10], for the above-mentioned parametric fault diagnosis of power electronics, the multi-device failure mode is added, and four kinds of algorithms are compared and analyzed. The examples show that all four diagnostic algorithms can achieve good diagnosis effect, and average fault diagnosis rate is over $90 \%$. In [11], BPNN is used to identify the circuit operating parameters and component fault characteristic parameters. According to the identification results, the fault characteristic parameters of the circuit are further obtained. The fault characteristic parameters of components and circuits are compared with their corresponding fault thresholds to determine whether A fault occurs to enable online fault diagnosis of components and circuits. In order to solve the fault diagnosis of closed-loop single-ended primary inductance converter, a new optimized deep belief network (DBN) is proposed in [12], which select the number of neurons in two hidden layers by Crow search algorithm (CSA) with training samples. Finally, it is classified by CSA-DBN. Compared with BPNN and support vector machine (SVM) fault diagnosis approaches, this approach has higher classification accuracy, but the optimized algorithm has a longer calculation time, and future research will focus on improving computational efficiency.

The parametric fault diagnosis approach based on neural network can learn, generalize and popularize from the sample, and has strong adaptive ability, but how to determine the appropriate network structure and scale, how to balance the convergence, rapidity and real-time of the algorithm and how to ensure the integrity and representativeness of the learning samples have yet to be further studied.

In the research of fuzzy reasoning approaches, a classifier fusion diagnosis approach based on fuzzy reasoning is proposed in [13]. The neural network and support vector machine classifier are used as two sub-classifiers of fuzzy inference input. The corresponding membership function is designed to fuzzify the output of the two classifiers, and the calculation is based on the pre-processed fuzzy transformation matrix to get the fusion output of the system. The simulation experiment and analysis of a Cuk circuit show that the obtained diagnostic method is effective and better than the diagnostic accuracy of any single subclassifier. At the same time, the approach is robust to random noise.

The advantage of parametric fault diagnosis based on fuzzy inference is that it can embed linguistic knowledge and approximate reasoning ability. However, compared with the traditional fault diagnosis theory and method, there is still immature. For example, effective methods have not been established to analyze and design fuzzy systems, mainly relying on expert experience and trial-and-error; fuzzy logic systems lack self-learning capabilities that are necessary in some highly demanding real-time fault diagnosis situations. 
In the pattern recognition approach, the aging of power insulated gate bipolar transistor (IGBT) modules is studied in [14]. The IGBT module of $1200 \mathrm{~V}-30 \mathrm{~A}$ specification has passed the power cycle aging test, aiming to highlight the external indicators of aging that can be measured online and in real time. Then, these indicators are diagnosed through manual training approaches based on pattern recognition. In [15], the fault identification approach based on Fisher discriminant analysis is studied and compared with the experimental results of RBF neural network identification. The results show that the diagnostic accuracy based on Fisher discriminant analysis is higher.

Pattern recognition theory is one of the most active topics in the field of artificial intelligence in recent years. Although it has obtained a lot of practical applications in image processing and speech recognition, its application in the field of parametric fault diagnosis is not enough.

\section{Signal-based fault diagnosis methods}

Signal-based fault diagnosis methods are to use the spectrum or wavelet technology to act on the signal model, extract relevant feature values for analysis, and diagnose the occurrence of the fault. This method is widely used in parametric fault diagnosis since it does not need to establish an accurate mathematical model and it is easy to implement in engineering. The schematic diagram is shown in Fig. 2. It is worth noting that when processing signals using methods such as spectral analysis, sometimes it is necessary to combine with the knowledge base to determine the location of the fault.

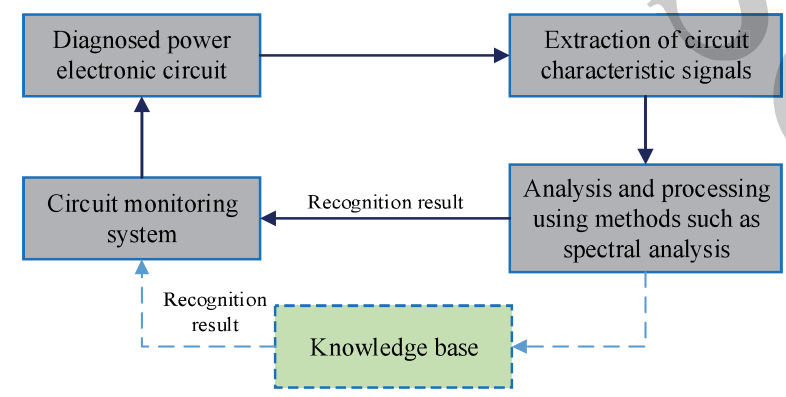

Fig. 2 Schematic of signal-based parametric fault diagnosis

A method for fault diagnosis of power electronic circuits based on wavelet analysis and Mahalanobis distance is proposed in [16], and it applied to DC-DC converter circuits. At the time of diagnosis, the unknown fault circuit data is measured, sampled and analyzed by wavelet, and the fault of the circuit to be tested can be judged by calculating the Mahalanobis distance between the unknown fault and the fault set. The algorithm is simple, the data storage amount and calculation amount are small and the application is convenient, and it is a very effective fault diagnosis method. [17] is based on the wavelet transform of the measurement signal, which sets the tolerance limit by statistical processing data obtained from a set of fault-free circuits. In wavelet analysis, two test metrics are introduced, one based on the normalized Euclidean distance discrimination factor and the other is the Mahalanobis distance discrimination factor. Both of these indicators rely on wavelet energy calculations. In [18], the discrete wavelet transform is used to filter the test signal, which is used as a filter bank to obtain the signal component sensitive to the change of the device parameters. At the same time, the coefficient of the polynomial approximating the component is calculated and used to develop learning vector of the feedforward neural network. Although the proposed algorithm can correctly identify single-parameter defects, the test conditions and the learningsensitive signals generated by the pre-processing process may adversely affect the selected parameters.

Signal-based fault diagnosis methods have lower requirements on the input signal and strong anti-noise ability. However, after signal processing, how to ensure the maximum information integrity of the original signal is always a problem worth studying.

\section{Other emerging fusion approachs}

In addition to the more commonly used methods described above, there are other methods or hybrid methods that are also used in power electronic parametric fault diagnosis.

A hybrid diagnostic framework based on hybrid bond graph (HBG) and genetic algorithm (GA) is proposed for multi-parameter fault diagnosis in power electronic circuits in [19]. The circuit is modeled by HBG modeling techniques and the GA approach is used to identify component failures. This approach is suitable for the diagnosis of single and multiple parameter faults. In [20], the capacitor aging process is manifested by increasing its ESR and it is based on the fact that its value should not exceed twice the initial value. In [21], the traditional SVM is added to the empirical mode decomposition for preprocessing and the principal component analysis is used to extract the characteristic parameters, and the diagnostic accuracy is greatly improved. There are lots of optimizations for SVM in [22], but it is excellent in structural fault diagnosis. There is no big breakthrough in the diagnosis of parametric faults. Therefore, the application of SVM to diagnose parametric faults is still to be discussed.

\section{THE MAIN PROBLEMS CURRENTLY FACING AND THE FUTURE DEVELOPMENT TREND}

At present, most of the fields of power electronic fault diagnosis focus on structural faults. There are still few researches on parametric faults and it is not comprehensive enough and not deep enough. Therefore, there are naturally many problems worthy of attention:

1) As the various methods described above still have some shortcomings, they need to continue to study further solutions.

2 ) In order to make the diagnosis result correct and reliable, it is always desirable to extract the fault feature quantity as much as possible [23]. However, as the number of fault features increases, it will inevitably cause certain difficulties for the actual test work, and there may be information redundancy between each fault quantity, increasing the complexity of the classifier model.

3 ) In the practical application of circuit fault diagnosis, online diagnostic technology has insufficient consideration of real-time requirements, and it is also worth considering[24].

Starting from the current problems in the field of parametric fault diagnosis of power electronic circuits, the future research focus can be divided into the following aspects: 
1) With the advent of the era of big data and the rise of artificial intelligence and deep learning, timely introduction of new theories and technologies into the field of fault diagnosis of power electronic circuits, forming a fault diagnosis technology with superior performance, which will be an important trend for the development of the field in the future.

2 ) As the complexity of the system increases, fault diagnosis techniques based on the integration of multiple data sources will also receive more attention. Using multisensor fusion methods or using a single sensor but using multiple information extraction methods to fuse different data sources, comprehensive evaluation can reflect various fault information, which can greatly improve fault coverage rate and accuracy rate of diagnosis results.

3 ) At present, studying the parametric fault of power electronics is to study a gradual process. How to perform effective real-time classification and detection when it reaches artificially set parameter fault range is a direction worthy of follow-up research.

\section{CONCLUSION}

In the field of power electronic fault diagnosis, although most of them are for structural fault research, practical problems such as aging of power electronic device parameters can not be ignored in recent years. This paper summarizes the widely used parametric fault diagnosis methods, including model-based, knowledge-based, signalbased and other emerging fusion methods, and explains correspondingly the research status, characteristics and deficiencies of these methods. Finally, the shortcomings of the current power electronics parametric fault diagnosis field and the future development direction were discussed. With the further study of parametric fault diagnosis, it will promote systematic and intelligent process strongly in the field of fault diagnosis.

\section{ACKNOWLEDGMENT}

This work was supported in part by the Technology Innovation Fund Support Project by the company of Kehua Hengsheng under Grant KHHS20170416.

\section{REFERENCES}

[1] A. Bindra and T. Keim, "Exciting advances in power electronics APEC 2018 divulges latest ddvances in magnetics, wide-bandgap devices, vehicle batteries, 3-D packaging, and more," IEEE Power Electronics Magazine, vol. 5, no. 2, pp. 49-55, June 2018.

[2] Y. Z. Zhang, J. Liu, J. F. Zhang and C. X. Wang, "Study on the development of renewable energy during 'post-subsidy period' in China," Electric Power, vol. 52, no. 4, pp. 1-7, April 2019

[3] H. Ma, X. Y. Mao, N. Zhang and D. H. Xu, "Parameter identification of power electronic circuits based on hybrid model," IEEE Power Electronics Specialists Conference(PESC), Recife, 2005

[4] X. J. Li and D. H. Zhou, "Fault diagnosis based on hybrid system model and filter for power electronic circuits," Journal of Northwest University ( Natural Science Edition), vol. 41, no. 3, June 2011.

[5] Q. Sun, Y. R.Wang, Y. Y. Jiang and Y. Wu, "On-line componentlevel soft fault diagnostics for power converters," Prognostics and System Health Management Conference (PHMC), Chengdu, 2017.

[6] G. M. Buiatti, J. A. Martín-Ramos, C. H. R. García, A. M. R. Amaral and A. J. M. Cardoso, "An online and noninvasive technique for the condition monitoring of capacitors in boost converters," IEEE
Transactions on Instrumentation and Measurement, vol. 59, no. 8, pp. 2134-2143, August 2010.

[7] X. S. Pu, T. H. Nguyen, D. C. Lee, K. B. Lee and J. M. Kim, "Fault diagnosis of dc-link capacitors in three-phase AC/DC PWM converters by online estimation of equivalent series resistance," IEEE Transactions on Industrial Electronics, vol. 60, no. 9, pp. 4118-4127, September 2013.

[8] S. Y. Yang, D. W. Xiang, A. Bryant, P. Mawby L. Ran and P. Tavner, "Condition monitoring for device reliability in power electronic converters: A review," IEEE Transactions on Power Electronics, vol. 25 , no. 11 , pp. 2734-2752, November 2010.

[9] M. Li, "Research on fault diagnosis technology for power electronic circuits," Nanjing University of Aeronautics and Astronautics, 2015.

[10] M. Li and Y. R. Wang, "Power electronic circuit soft fault diagnosis methods comparative analysis," Electronic Measurement Technology, vol. 38, no. 7, pp. 110-114, July 2015.

[11] Y. Wu, Y. R. Wang, Y. Chen and Y. Y. Jiang, "Parametric fault diagnosis method of power electronic circuit based on BPNN," Journal of Electronic Measurement and Instrument, vol. 26, no. 5, pp. 458-462, May 2012

[12] Q. Sun, Y. R. Wang and Y. Y. Jiang, "A novel fault diagnostic approach for DC-DC converters based on CSA-DBN," IEEE Access, vol. 6, pp.6273-6285, 2018.

[13] J. Cui, and Y. R. Wang, "Testing the parametric faults of power electronic circuits applying classifiers fusion method based on fuzzy inference," Proceedings of the CSEE, vol. 29, no. 18, pp. 54-59, June 2009.

[14] A. Oukaour, B. Tala-Ighil, B. Pouderoux, M. Tounsi, M. BouarroudjBerkani, et al, "Ageing defect detection on IGBT power modules by artificial training methods based on pattern recognition," Microelectronics Reliability, vol. 52, no. 2, pp. 386-391, 2011.

[15] S. Y. Jiang, "The research of fault diagnosis and fault prognostics method for power electronic circuits," Hunan University, 2016.

[16] Y. Y. Sheng and Z. Z. Hu, "Fault diagnosis of power electronic circuit based on wavelet and mahalanobis distance," Electronic Measurement Technology, vol. 36, no. 2, pp. 108-112, February 2013.

[17] A. D. Spyronasios, M. G. Dimopoulos and A. A. Hatzopoulos, "Wavelet analysis for the detection of parametric and catastrophic faults in mixed-signal circuits," IEEE Transactions on Instrumentation and Measurement, vol. 60, no. 6, pp. 2025-2038, July 2011.

[18] A. Kuczy'nski, "Parametric faults detection in analog circuits using polynomial coefficients in NN learning," International Conference on Signals and Electronic Systems, September 2010.

[19] Y. Wu, Y. R. Wang, Y. Y. Jiang and Q. Sun, "Multiple parametric faults diagnosis for power electronic circuits based on hybrid bond graph and genetic algorithm," Measurement, vol. 92, pp. 365-381, 2016.

[20] A. M. R. Amaral, and A.J.M. Cardoso, "On-line fault detection of aluminium electrolytic capacitors, in step-down DC-DC converters, using input current and output voltage ripple," IET Power Electronics, vol. 5, Iss. 3, pp. 315-322, 2012.

[21] B. B. Miao, Y. X. Shen, D. H. Wu and Z. P. Zhao, "Three level inverter fault diagnosis using EMD and support vector machine approach," 2017 12th IEEE Conference on Industrial Electronics and Applications (ICIEA), Siem Reap, 2018, pp. 1595-1598.

[22] T. Z. Wang, H. Xu, J. G. Han, E. Elbouchikhi and M. E. H. Benbouzid, "Cascaded H-bridge multilevel inverter system fault diagnosis using a PCA and multi-class relevance vector machine approach," IEEE Transactions on Power Electronics, vol. 30, no. 12, pp. 7006-7018, December 2015.

[23] J. S. Zhang, B. Y. Song and H. D. Zhao, "A new approach to fault diagnosis of three-phase full-bridge rectifier with integrated feature extraction" 2017 Chinese Automation Congress (CAC), Jinan, 2017, pp. 6817-6821.

[24] H. Givi, E. Farjah and T. Ghanbari, "A comprehensive monitoring system for online fault diagnosis and aging detection of non-isolated DC-DC converters' components" IEEE Transactions on Power Electronics, vol. 34, no. 7, pp. 6858-6875, October 2018. 